\title{
Desigualdades sociais em saúde e na utilização dos serviços de saúde entre os idosos na América Latina
}

\author{
Kenya Valéria Micaela de Souza Noronha1 e Mônica Viegas Andrade1
}

Como citar

Noronha KVMS, Andrade MV. Desigualdades sociais em saúde e na utilização dos serviços de saúde entre os idosos na América Latina. Rev Panam Salud Publica. 2005;17(5/6):410-8.

RESUMO Objetivo. Verificar se existe desigualdade social em saúde e na utilização dos serviços de saúde entre os idosos de Buenos Aires, São Paulo, Santiago, Havana, Cidade do México e Montevidéu. Métodos. Foram utilizados dados do Projeto Saúde, Bem-Estar e Envelhecimento na América Latina e Caribe ( $S A B E$ ). Para verificar a presença de desigualdade social em saúde, utilizouse o modelo probit ordenado, tendo como variáveis dependentes os seguintes indicadores do estado de saúde: atividades de vida diária, atividades instrumentais de vida diária, mobilidade física e estado de saúde auto-avaliado. Para mensurar a desigualdade social na utilização dos serviços de saúde, duas especificações foram testadas: uma para os serviços médicos ambulatoriais (se consultou o médico e quantas vezes consultou) e outra para os serviços de internação hospitalar (se foi internado). Para os cuidados ambulatoriais, estimamos o modelo hurdle binomial negativo, e para os serviços de internação hospitalar, utilizamos um modelo logit.

Resultados. Os principais resultados sugerem a presença de desigualdade social em saúde em todas as cidades, favorável aos grupos socioeconômicos privilegiados. Essa diferença foi menor em Havana, Buenos Aires e Montevidéu e mais acentuada em São Paulo e na Cidade do México. Quanto à utilização dos serviços de saúde, detectamos a presença de desigualdade no uso de serviços ambulatoriais em Santiago, na Cidade do México e em São Paulo. Em Santiago e na Cidade do México, quanto maior o nível educacional, maior o número esperado de consultas. Em São Paulo, o oposto foi observado. Para os serviços de internação hospitalar, a presença de desigualdade foi detectada apenas em São Paulo, favorecendo os grupos mais escolarizados. Conclusão. Os presentes resultados refletem, em certa medida, as características socioeconômicas e demográficas dos países de cada cidade estudada. As cidades dos países que apresentam os piores indicadores sociais (elevada desigualdade de renda e baixo índice de desenvolvimento humano) tenderam a apresentar as maiores desigualdades em saúde e na utilização dos serviços de saúde.

Palavras-chave Escolaridade, sistema de saúde.

1 Universidade Federal de Minas Gerais (UFMG), Centro de Desenvolvimento e Planejamento Regional (CEDEPLAR), Belo Horizonte (MG), Brasil. Enviar correspondência para Kenya de Souza Noronha no seguinte endereço: Rua Curitiba 832, $9^{\circ}$ andar, Centro, CEP 30170-120, Belo Horizonte, MG, Brasil. Telefone: +55-31-3279-9081; e-mail: kenya@ cedeplar.ufmg.br e mviegas@cedeplar. ufmg.br
Nas últimas décadas, os países da América Latina têm vivenciado um rápido processo de envelhecimento devido à redução das taxas de mortalidade e, principalmente, das taxas de fecundidade. Para os países analisados no presente trabalho - Argentina, Bra- sil, Chile, Cuba, México e Uruguaias projeções indicam uma participação crescente da população idosa, que deverá chegar, em 2030, a 13, 12, 15, 21, 11 e $15 \%$, respectivamente (1). Essas mudanças no padrão demográfico acarretam importantes implicações para as 
políticas sociais, especialmente para as políticas de saúde. Nas economias mais desenvolvidas, como o envelhecimento populacional foi gradativo, foi possível promover a organização dos sistemas de previdência e de saúde de forma a acomodar o aumento da demanda nesses setores, o que não ocorreu nos países em desenvolvimento (2).

Existem ainda poucos estudos que buscam investigar a presença de desigualdade social em saúde e na utilização dos serviços médicos entre os idosos, sobretudo análises comparativas entre os países menos desenvolvidos. A maior parte desses trabalhos foi realizada para economias mais avançadas e se constitui em uma extensão da literatura que enfoca todos os grupos de idade e a população em idade ativa. Nesses países desenvolvidos, a evidência empírica constata a presença de desigualdades sociais em saúde e na utilização dos serviços médicos favoráveis aos idosos que pertencem a grupos sociais privilegiados (3-12). $\mathrm{Na}$ América Latina, os estudos existentes avaliam a presença de desigualdades considerando todos os grupos etários e indicam que, quanto melhores as con- dições sociais dos indivíduos ou das localidades, melhor é o estado de saúde e o acesso aos serviços (13-21).

Como os idosos apresentam um estado de saúde mais vulnerável, e como a fração da renda gasta com saúde é maior para esse grupo populacional, a investigação acerca da presença dessas desigualdades é um subsídio importante para a definição de políticas públicas e sociais na região.

O objetivo deste trabalho foi verificar a existência de desigualdade social em saúde e na utilização dos serviços de atenção à saúde entre os idosos em seis cidades da América Latina: Buenos Aires (Argentina), São Paulo (Brasil), Santiago (Chile), Havana (Cuba), Cidade do México (México) e Montevidéu (Uruguai).

\section{MATERIAIS E MÉTODOS}

Foram utilizados os dados do Projeto Saúde, Bem-Estar e Envelhecimento na América Latina e Caribe (SABE) (22), realizado em 2000, abrangendo a área urbana das seguintes cidades: Buenos Aires (Argentina), Bridgetown (Barba- dos), São Paulo (Brasil), Santiago (Chile), Havana (Cuba), Cidade do México (México) e Montevidéu (Uruguai). Os dados de Bridgetown não foram considerados, tendo em vista a indisponibilidade de uma base de dados completa até o momento da realização deste estudo.

Para compor a amostra, os indivíduos com 60 anos ou mais foram randomicamente selecionados (2). A amostra foi construída em cada cidade de forma a permitir comparabilidade, sendo estratificada por grupos etários com sobreamostragem da população acima de 80 anos (e da população acima de 75 anos em São Paulo) $(23,24)$.

\section{Variáveis dependentes}

Para avaliar a presença de desigualdade social em saúde, estimamos um modelo probit ordenado (25).

As variáveis dependentes compreendem quatro indicadores do estado de saúde-atividades de vida diária (AVD), atividades instrumentais de vida diária (AIVD), mobilidade física e estado de saúde auto-avaliado (tabela 1). A escolha de mais de um indi-

TABELA 1. Variáveis dependentes do modelo probit ordenado, estudo sobre desigualdades sociais em saúde e na utilização dos serviços de saúde entre os idosos de seis cidades da América Latina, 2000

\begin{tabular}{|c|c|c|}
\hline Variáveis dependentes & Descrição & Valor \\
\hline \multicolumn{3}{|l|}{ Aspectos da funcionalidade física } \\
\hline Atividades de vida diária & $\begin{array}{l}\text { Cruzar o quarto, vestir-se, tomar banho, comer, } \\
\text { levantar-se e deitar na cama, ir ao banheiro }\end{array}$ & $\begin{array}{l}\text { De } 0 \text { (indivíduo saudável, capaz de realizar } \\
\text { todas as atividades) a } 6 \text { (indivíduo com } \\
\text { dificuldade para realizar todas as atividades) }\end{array}$ \\
\hline Atividades instrumentais de vida diária & $\begin{array}{l}\text { Preparar comida, manusear o próprio dinheiro, } \\
\text { sair, comprar alimentos, telefonar, realizar } \\
\text { tarefas domésticas leves, realizar tarefas } \\
\text { domésticas pesadas e tomar medicamentos }\end{array}$ & $\begin{array}{l}\text { De } 0 \text { (indivíduo saudável, capaz de realizar } \\
\text { todas as atividades) a } 8 \text { (indivíduo com } \\
\text { dificuldade para realizar todas as atividades) }\end{array}$ \\
\hline \multirow[t]{4}{*}{ Mobilidade física } & $\begin{array}{l}\text { Consegue correr um quilômetro e meio } \\
\text { Não consegue correr um quilômetro, mas }\end{array}$ & 0 \\
\hline & $\begin{array}{l}\text { consegue caminhar várias ruas } \\
\text { Não consegue caminhar várias ruas, mas }\end{array}$ & 1 \\
\hline & consegue caminhar uma rua & 2 \\
\hline & Não consegue caminhar uma rua & 3 \\
\hline \multicolumn{3}{|l|}{ Aspectos mais amplos do estado de saúde } \\
\hline \multirow[t]{5}{*}{ Estado de saúde auto-avaliado } & Excelente & 0 \\
\hline & Muito bom & 1 \\
\hline & Bom & 2 \\
\hline & Regular & 3 \\
\hline & Ruim & 4 \\
\hline
\end{tabular}


cador permite considerar as várias dimensões do estado de saúde, além de verificar a robustez dos resultados. Todas essas medidas são variáveis categóricas ordenadas, não-binárias, onde o valor mínimo e o máximo representam, respectivamente, o melhor e o pior estado de saúde. A estimação do modelo probit ordenado permite que a probabilidade de ocorrência de cada categoria seja estimada sem a necessidade de se estabelecer um ponto de corte para classificar os indivíduos como doentes ou saudáveis.

As variáveis de saúde utilizadas neste trabalho referem-se a medidas auto-reportadas, obtidas através de entrevista domiciliar. Reconhecemos que essa é uma limitação, uma vez que as respostas dos indivíduos estão condicionadas ao estado de saúde das demais pessoas da comunidade e às condições do ambiente no qual estão inseridos, o que afeta sua percepção a respeito de seu próprio estado de saúde. Neste trabalho, como estamos realizando uma análise comparativa entre cidades de diferentes países, que são bastante heterogêneas, as diferenças no estado de saúde do idoso entre as localidades podem refletir tanto um estado de saúde precário como também as características do contexto em que o idoso está inserido. Uma forma de superar o problema é a utilização de instrumentos, a fim de tornar as respostas comparáveis entre as diferentes localidades analisadas (26). Infelizmente, não dispomos desses instrumentos na base de dados utilizada.

Para verificar a presença de desigualdade social na utilização dos serviços de saúde, duas especificações foram testadas: uma para os serviços médicos ambulatoriais e outra para os serviços de internação hospitalar. Para os cuidados ambulatoriais, estimamos o modelo hurdle binomial negativo, o qual considera que a utilização dos serviços ocorre em duas etapas. $\mathrm{Na}$ primeira, a partir de um modelo de regressão logística, estima-se a probabilidade de o indivíduo consultar o médico, e na segunda, utilizando um modelo binomial negativo truncado ao zero, estima-se o número esperado de consultas para os indivíduos com utilização positiva. Para os serviços de internação hospitalar, devido ao número reduzido de observações, a análise restringe-se à probabilidade de o indivíduo ser internado, ou seja, à primeira etapa do modelo. As variáveis dependentes no modelo ambulatorial foram "consultou o médico nos últimos 4 meses" ( $\operatorname{sim}=1$; não $=0) \mathrm{e}$ "quantas vezes consultou o médico" (valores inteiros maiores ou iguais a 1). No modelo de internação, a variável dependente foi "internação nos últimos 4 meses" $(\operatorname{sim}=1$; não $=0)$.

\section{Variáveis independentes}

A escolha das variáveis independentes baseou-se no modelo de demanda por saúde desenvolvido por Grossman (27). De acordo com esse modelo, cada indivíduo possui uma função de produção que transforma insumos em estoque de capital de saúde. Esses insumos são o tempo dos indivíduos e a demanda por cuidados médicos. Além dos insumos, o estoque de saúde depende da dotação inicial de saúde, determinada geneticamente; de certas características do ambiente em que os indivíduos vivem, como a presença de saneamento básico e a oferta de serviços de saúde; das características da família; e do nível de escolaridade do indivíduo. De um modo geral, o modelo estabelece que o estoque de saúde é influenciado pelas escolhas que o indivíduo faz ao longo de sua vida, que, por sua vez, são realizadas de acordo com a função de utilidade de cada indivíduo.

A tabela 2 apresenta as variáveis independentes incluídas nos modelos de análise. Para o modelo que analisa a utilização dos serviços de saúde, foram incluídos, além das variáveis listadas na tabela 2, indicadores do estado de saúde e do acesso aos serviços de saúde, medidos pela presença de seguro-saúde. No primeiro grupo de indicadores, foram considerados o número de doenças crônicas ou agudas (hipertensão, diabetes, câncer, problemas cardíacos, derrame, asma, reumatismo e problemas psiquiátricos), o estado de saúde auto-avaliado, a mo- bilidade física, as AVD e as AIVD. No segundo grupo de indicadores (indicadores de acesso aos serviços), foram consideradas, nos modelos estimados para Buenos Aires e para a Cidade do México, as variáveis binárias "possui seguro social de saúde", "possui plano privado de saúde" e "não possui plano ou seguro-saúde"; e, para São Paulo, Santiago e Montevidéu, a variável "possui plano privado ou segurosaúde". No modelo estimado para Havana, a variável de acesso aos serviços não foi incluída, pois todos os serviços de saúde nessa cidade são financiados e providos pelo Estado.

No presente estudo, a medida socioeconômica utilizada foi a variável escolaridade, que classifica a amostra em dois grupos: idosos com 7 anos ou menos de estudo ou com 8 anos ou mais (tabela 2). ${ }^{2}$ Essa variável procura medir pelo menos dois aspectos socioeconômicos relacionados com o nível de saúde individual.

O primeiro refere-se ao comportamento dos indivíduos, que está condicionado ao conjunto de informações que eles possuem. As pessoas mais escolarizadas tendem a adotar hábitos de vida mais saudáveis e a procurar mais os serviços médicos, especialmente os cuidados preventivos, uma vez que, ao possuírem mais informações sobre as doenças e os possíveis tratamentos ou diagnósticos, tendem a valorizar de forma diferenciada o cuidado com a saúde. Nesse sentido, o nível cultural do indivíduo afeta a eficiência da função de produção de saúde. $\mathrm{O}$ segundo refere-se à disponibilidade de recursos para investir na saúde, uma vez que, quanto maior o nível de escolaridade, maior tende a ser o nível de renda e, conseqüentemente, melhores as condições de vida e de acesso aos serviços de saúde.

Uma forma de separar esses dois aspectos associados à escolaridade é

\footnotetext{
2 O modelo foi estimado também a partir da escolaridade do chefe de família. Os resultados, contudo, não foram substancialmente diferentes. A construção da variável de escolaridade baseou-se no algoritmo desenvolvido pela Dra. Laura Lídia Rodríguez Wong, do Departamento de Demografia do Centro de Desenvolvimento e Planejamento Regional (CEDEPLAR) da Universidade Federal de Minas Gerais (UFMG)
} 
TABELA 2. Variáveis independentes incluídas nos modelos de avaliação das desigualdades sociais em saúde e na utilização dos serviços de saúde entre os idosos de seis cidades da América Latina, 2000

\begin{tabular}{|c|c|}
\hline Variáveis independentes & Categorias de resposta \\
\hline \multicolumn{2}{|l|}{ Medida socioeconômica } \\
\hline \multirow[t]{2}{*}{ Escolaridade } & $\leq 7$ anos de estudo (categoria de referência) \\
\hline & 8 anos ou mais \\
\hline \multicolumn{2}{|l|}{ Variáveis demográficas } \\
\hline \multirow{2}{*}{ Sexo } & Homem $=1$ \\
\hline & Mulher $=0$ \\
\hline \multirow[t]{6}{*}{ Idade (anos) } & 60 a 64 \\
\hline & 65 a 69 \\
\hline & 70 a 74 \\
\hline & 75 a 79 \\
\hline & 80 a 84 \\
\hline & 85 e mais (categoria de referência) \\
\hline \multicolumn{2}{|l|}{ Rede de apoio familiar } \\
\hline \multirow[t]{5}{*}{ Composição familiar } & Mora sozinho (categoria de referência) \\
\hline & Mora apenas com o cônjuge \\
\hline & $\begin{array}{l}\text { Mora com os filhos solteiros, } \\
\text { independentemente de o cônjuge estar } \\
\text { presente ou não }\end{array}$ \\
\hline & Mora com pelo menos um filho casado \\
\hline & $\begin{array}{l}\text { Mora com outros membros da família, como } \\
\text { netos, sobrinhos }\end{array}$ \\
\hline Número de pessoas no domicílio & Variável discreta \\
\hline \multirow[t]{4}{*}{ Estado conjugal } & Casado \\
\hline & Desquitado ou separado \\
\hline & Viúvo \\
\hline & Solteiro (categoria de referência) \\
\hline \multicolumn{2}{|c|}{ Dotação inicial de saúde (primeiros 15 anos de vida) } \\
\hline \multirow[t]{3}{*}{ Estado de saúde auto-avaliado } & Excelente \\
\hline & Bom \\
\hline & Ruim (categoria de referência) \\
\hline \multirow[t]{3}{*}{ Condição socioeconômica da família } & Boa \\
\hline & Regular \\
\hline & Ruim (categoria de referência) \\
\hline \multirow[t]{2}{*}{ Fome nos primeiros 15 anos de vida } & Teve fome $=1$ \\
\hline & Não teve fome $=0$ \\
\hline \multirow[t]{2}{*}{ Local de residência } & Rural = 1 \\
\hline & Urbano $=0$ \\
\hline \multicolumn{2}{|l|}{ Hábitos de vida } \\
\hline \multirow[t]{3}{*}{ Fumo } & Fumante \\
\hline & Já fumou e atualmente não fuma mais \\
\hline & Nunca fumou \\
\hline \multirow[t]{2}{*}{ Consumo de bebida alcoólica } & Mais do que três copos por dia \\
\hline & Menos do que três copos por dia \\
\hline \multirow[t]{2}{*}{ Exercício físico } & Pratica \\
\hline & Não pratica \\
\hline
\end{tabular}

Fonte dos dados: Projeto Saúde, Bem-Estar e Envelhecimento na América Latina e Caribe (SABE) (22).

a inclusão da variável de rendimento, que representa o fluxo de renda corrente dos indivíduos e mede a disponibilidade de recursos para investir em saúde. Apesar de estar presente na pesquisa, essa variável não pôde ser incluída devido ao elevado número de respostas ausentes.
Além das variáveis de fluxo, pode-se controlar a condição socioeconômica através das variáveis de estoque de riqueza, que representam a renda permanente. Essa possibilidade foi testada através da inclusão dos itens "posse de automóvel" e "posse de casa própria".

\section{Caracterização dos países}

A análise realizada neste trabalho compreende cidades de países diferenciados em termos de padrão de envelhecimento, nível de desenvolvimento econômico e desenho institucional dos sistemas de saúde. Essas diferenças podem acarretar padrões distintos de desigualdades sociais em saúde e na utilização dos serviços de saúde.

Considerando-se as características do processo de transição demográfica, podemos classificar os seis países em três grupos (28): o primeiro compreende a Argentina, Cuba e o Uruguai, cujo processo é precoce em relação às demais economias da região e que se destacam por apresentar os melhores indicadores socioeconômicos (tabela 3) (29-35). No segundo grupo está o México, cujo processo de transição foi tardio. O Brasil e o Chile se classificam no grupo de transição intermediária. Ressalta-se que essa tipologia foi desenvolvida para os países, e não para as cidades. Nesse sentido, este trabalho utiliza informações sobre a média do país como uma aproximação para as cidades amostradas. Apesar de apresentarem um perfil demográfico e de desenvolvimento mais avançado do que aquele observado no âmbito nacional, as cidades acompanham o processo observado no país, uma vez que estão inseridas nesse padrão médio. Como a análise foi realizada para a principal cidade de cada país, mesmo que estejam em um nível mais avançado do que a média nacional, é razoável supor que essas cidades mantêm entre si a mesma posição relativa no processo de transição demográfica observada entre os respectivos países.

Com relação às características dos sistemas de saúde, dos seis países analisados, cinco apresentam sistemas mistos de saúde, mas a participação de cada um dos setores, privado e público, é bastante diferenciada. Uma possível tipologia classifica os países segundo a principal fonte de financiamento, variando de uma cobertura com predomínio de recursos privados até uma cobertura totalmente pública. De acordo com essa classificação, os países seriam ordenados da seguinte 
TABELA 3. Indicadores socioeconômicos de seis países e cidades incluídos no Projeto Saúde, Bem-Estar e Envelhecimento na América Latina e Caribe (SABE), 2000

\begin{tabular}{|c|c|c|c|}
\hline & $\begin{array}{l}\text { Índice } \\
\text { de Gini }\end{array}$ & $\begin{array}{c}\text { Taxa de analfabetismo } \\
\text { de adultos }{ }^{b}\end{array}$ & $\mathrm{IDH}^{\mathrm{b}}$ \\
\hline \multicolumn{4}{|l|}{ Países } \\
\hline Argentina & 47,02 & 3,2 & 0,844 \\
\hline Brasil & $65,00^{b}$ & 14,8 & 0,757 \\
\hline Chile & 56,02 & 4,2 & 0,831 \\
\hline Cuba & $\ldots$ & 3,3 & 0,795 \\
\hline México & 52,76 & 8,6 & 0,796 \\
\hline Uruguai & 42,09 & 2,3 & 0,831 \\
\hline \multicolumn{4}{|l|}{ Cidades $^{c}$} \\
\hline Buenos Aires $^{b}$ & $\ldots$ & 2,4 & 0,892 \\
\hline São Paulob & 62,00 & 4,9 & 0,841 \\
\hline Havana $^{b}$ & $\ldots$ & $\ldots$ & 0,7278 \\
\hline Cidade do Méxicob & $\ldots$ & 2,90 & 0,891 \\
\hline Montevidéu & $\ldots$ & $\ldots$ & 0,848 \\
\hline
\end{tabular}

forma: Uruguai, Chile, Brasil, Argentina, México e Cuba.

Considerando o total da população, observamos que $55 \%$ dos indivíduos no Uruguai são cobertos pelo setor privado, contra $35 \%$ no Chile e $25 \%$ no Brasil. Em Cuba, 100\% da população recebe atendimento no setor público. $\mathrm{Na}$ Argentina e no México, a maior parte da população é atendida pelo sistema de seguridade social (52 e 49\%, respectivamente) (36-38). Essas diferenças no grau de cobertura por tipo de financiamento resultam das diferenças na organização da oferta dos serviços de saúde. No Brasil, Chile e Uruguai, o sistema de saúde é composto por dois setores: público e privado. $\mathrm{O}$ atendimento no setor público pode ocorrer através da cobrança de alguma taxa, que varia segundo a renda, como no caso do Chile e Uruguai, ou gratuitamente, como no Brasil. $\mathrm{O}$ atendimento no setor privado é, em sua maioria, realizado a partir da compra de planos ou seguros de saúde, cuja adesão pode ser voluntária ou compulsória. Na Argentina e no México, a organização do sistema de saúde é mais segmentada. Além dos setores público e privado, a oferta dos serviços ocorre também através do sistema de seguridade social. Esse sis- tema está vinculado ao mercado de trabalho, e a adesão é compulsória. Dos países analisados, Cuba é o único onde o Estado assume totalmente a responsabilidade de organizar e financiar a oferta dos serviços, com cobertura de $100 \%$ da população.

\section{RESULTADOS}

\section{Desigualdade social em saúde}

Os resultados apontam a presença de desigualdade social em saúde a favor dos grupos de maior escolaridade em todas as cidades analisadas. Considerando o estado de saúde autoavaliado como a variável dependente, a diferença entre os dois grupos de escolaridade foi mais acentuada na $\mathrm{Ci}$ dade do México, em Santiago e em São Paulo, e menor em Havana, em Buenos Aires e em Montevidéu (tabela 4). Na Cidade do México, a chance de o idoso relatar um estado de saúde excelente foi 4,42 vezes maior para os que tinham mais de 7 anos de estudo em relação aos que tinham menor nível de instrução. Por outro lado, em Havana, essa diferença foi igual a 1,8.

No caso das AIVD, em todos os países, a probabilidade de o idoso ser sau- dável (realizar sozinho todas essas atividades) foi maior entre os que tinham mais de 7 anos de estudo. Quando consideramos a probabilidade de o idoso relatar pelo menos uma incapacidade, essa relação se inverteu, ou seja, a probabilidade se tornou mais elevada entre os que tinham menor nível de instrução, aumentando de acordo com o número de atividades que o idoso não conseguia realizar (tabela 4). As cidades que apresentaram as maiores desigualdades foram São Paulo, Havana e a Cidade do México, onde a probabilidade de ser saudável foi, respectivamente, $28,36 \%, 21,07 \%$ e $20,43 \%$ maior para os mais escolarizados em comparação aos que tinham menos de 7 anos de estudo. A realização das AIVD depende dos padrões culturais e sociais do meio em que o indivíduo está inserido, os quais, por sua vez, são determinados, em grande medida, pelas condições socioeconômicas. É possível que os idosos de maior escolaridade tenham menos necessidade de realizar parte dessas atividades, seja porque têm quem as realize em seu lugar, seja porque as condições para realizar essas tarefas são mais favoráveis.

Com relação às $\mathrm{AVD}$, constatamos uma desigualdade social em saúde mais elevada em Santiago, em São Paulo e na Cidade do México. A probabilidade de o idoso ser saudável segundo esse critério foi $16,49 \%$ maior para os que tinham mais de 7 anos de estudo em Santiago em comparação com aqueles menos escolarizados (tabela 4). Em São Paulo e na Cidade do México, essa diferença foi de cerca de $13 \%$. Essa relação se inverteu quando analisamos a probabilidade de o idoso não conseguir realizar pelo menos uma dessas atividades. Nesse caso, a chance de apresentar alguma restrição de atividade foi mais elevada entre os idosos com menor nível de instrução, aumentando monotonicamente com o número de incapacidades.

Para o grau de mobilidade física, detectamos a presença de desigualdade social em saúde a favor dos mais escolarizados em todas as cidades, exceto em Montevidéu. Essa desigualdade foi mais acentuada em Buenos Aires e na 
TABELA 4. Valores médios preditos da probabilidade de ocorrência de cada condição de saúde dos idosos de seis cidades latino-americanas conforme a escolaridade ${ }^{\mathrm{a}}$

\begin{tabular}{|c|c|c|c|c|c|c|c|c|c|c|c|c|}
\hline & \multicolumn{12}{|c|}{ Probabilidade de ocorrência } \\
\hline & \multicolumn{6}{|c|}{$\leq 7$ anos de estudo } & \multicolumn{6}{|c|}{$>7$ anos de estudo } \\
\hline & $\begin{array}{l}\text { Buenos } \\
\text { Aires }\end{array}$ & Havana & $\begin{array}{l}\text { São } \\
\text { Paulo }\end{array}$ & $\begin{array}{c}\text { Cidade } \\
\text { do México }\end{array}$ & Santiago & Montevidéu & $\begin{array}{c}\text { Buenos } \\
\text { Aires }\end{array}$ & Havana & $\begin{array}{l}\text { São } \\
\text { Paulo }\end{array}$ & $\begin{array}{c}\text { Cidade } \\
\text { do México }\end{array}$ & Santiago & Montevidéu \\
\hline \multicolumn{13}{|l|}{$\begin{array}{l}\text { Estado de saúde } \\
\text { auto-avaliado }\end{array}$} \\
\hline Excelente & 0,031 & 0,014 & 0,037 & 0,014 & 0,008 & 0,043 & $0,074^{b}$ & $0,025^{b}$ & $0,111^{b}$ & $0,062^{b}$ & $0,03^{b}$ & $0,105^{b}$ \\
\hline Regular & 0,329 & 0,516 & 0,480 & 0,548 & 0,437 & 0,355 & $0,22^{b}$ & $0,473^{b}$ & $0,315^{b}$ & $0,443^{b}$ & $0,362^{b}$ & $0,239^{b}$ \\
\hline Ruim & 0,067 & 0,154 & 0,088 & 0,205 & 0,246 & 0,081 & $0,028^{b}$ & $0,104^{b}$ & $0,026^{b}$ & $0,07^{b}$ & $0,103^{b}$ & $0,034^{b}$ \\
\hline \multicolumn{13}{|l|}{ Número de AIVDC } \\
\hline 0 & 0,694 & 0,688 & 0,670 & 0,734 & 0,685 & 0,806 & $0,817^{d}$ & $0,833^{b}$ & $0,860^{b}$ & $0,884^{b}$ & $0,824^{b}$ & $0,885^{e}$ \\
\hline 1 & 0,114 & 0,115 & 0,114 & 0,112 & 0,101 & 0,094 & $0,082^{d}$ & $0,075^{b}$ & $0,064^{b}$ & $0,060^{\mathrm{b}}$ & $0,069^{b}$ & $0,062^{\mathrm{e}}$ \\
\hline 2 & 0,066 & 0,060 & 0,067 & 0,060 & 0,064 & 0,042 & $0,041^{d}$ & $0,034^{b}$ & $0,031^{b}$ & $0,026^{b}$ & $0,039^{b}$ & $0,025^{e}$ \\
\hline 3 & 0,033 & 0,039 & 0,048 & 0,037 & 0,043 & 0,026 & $0,019^{d}$ & $0,020^{b}$ & $0,019^{b}$ & $0,014^{b}$ & $0,023^{b}$ & $0,014^{e}$ \\
\hline 8 & 0,013 & 0,019 & 0,019 & 0,010 & 0,013 & 0,003 & $0,004^{d}$ & $0,005^{b}$ & $0,003^{b}$ & $0,002^{b}$ & $0,004^{b}$ & $0,001^{e}$ \\
\hline \multicolumn{13}{|l|}{ Número de $A V D^{c}$} \\
\hline 0 & 0,800 & 0,777 & 0,799 & 0,813 & 0,746 & 0,802 & $0,903^{e}$ & $0,873^{b}$ & $0,91^{d}$ & $0,921^{b}$ & $0,869^{b}$ & $0,884^{e}$ \\
\hline 1 & 0,098 & 0,102 & 0,097 & 0,094 & 0,088 & 0,104 & $0,056^{e}$ & $0,067^{b}$ & $0,051^{d}$ & $0,047^{b}$ & $0,055^{b}$ & $0,067^{e}$ \\
\hline 2 & 0,041 & 0,046 & 0,048 & 0,035 & 0,076 & 0,053 & $0,02^{\mathrm{e}}$ & $0,026^{b}$ & $0,021^{d}$ & $0,014^{b}$ & $0,04^{b}$ & $0,03^{e}$ \\
\hline 3 & 0,023 & 0,022 & 0,017 & 0,024 & 0,030 & 0,018 & $0,009^{e}$ & $0,011^{\mathrm{b}}$ & $0,006^{d}$ & $0,009^{b}$ & $0,014^{b}$ & $0,009^{e}$ \\
\hline 4 & 0,015 & 0,018 & 0,011 & 0,012 & 0,018 & 0,015 & $0,005^{\mathrm{e}}$ & $0,009^{b}$ & $0,004^{d}$ & $0,004^{b}$ & $0,008^{b}$ & $0,007^{e}$ \\
\hline 5 & 0,019 & 0,018 & 0,014 & 0,009 & 0,016 & 0,005 & $0,006^{e}$ & $0,008^{b}$ & $0,004^{d}$ & $0,003^{b}$ & $0,006^{b}$ & $0,002^{\mathrm{e}}$ \\
\hline 6 & 0,004 & 0,018 & 0,015 & 0,012 & 0,026 & 0,003 & $0,001^{\mathrm{e}}$ & $0,006^{b}$ & $0,004^{d}$ & $0,003^{b}$ & $0,008^{b}$ & $0,001^{\mathrm{e}}$ \\
\hline \multicolumn{13}{|l|}{ Mobilidade física } \\
\hline Sem limitação & 0,033 & 0,097 & 0,091 & 0,237 & 0,127 & 0,077 & $0,077^{e}$ & $0,191^{b}$ & $0,170^{d}$ & $0,390^{b}$ & $0,208^{b}$ & $0,136^{f}$ \\
\hline Pequena limitação & 0,587 & 0,520 & 0,616 & 0,429 & 0,485 & 0,547 & $0,707^{e}$ & $0,577^{b}$ & $0,657^{d}$ & $0,418^{b}$ & $0,528^{b}$ & $0,591^{\dagger}$ \\
\hline Média limitação & 0,227 & 0,233 & 0,172 & 0,215 & 0,243 & 0,248 & $0,153^{e}$ & $0,162^{b}$ & $0,115^{d}$ & $0,140^{\mathrm{b}}$ & $0,184^{b}$ & $0,194^{\dagger}$ \\
\hline
\end{tabular}

Fonte dos dados básicos: Projeto Saúde, Bem-Estar e Envelhecimento na América Latina e Caribe (SABE) (22).

a Os níveis de significância correspondem aos resultados dos coeficientes estimados para o grupo com mais de 7 anos de escolaridade, tendo como categoria de referência os idosos com

7 anos ou menos de estudo.

b Significativo a $1 \%$.

${ }^{c}$ AIVD = atividades instrumentais de vida diária; $A V D=$ atividades de vida diária

d Significativo a $10 \%$.

e Significativo a $5 \%$.

f Não significativo.

Cidade do México. Nessas duas cidades, a chance de o idoso relatar incapacidade severa (não consegue caminhar uma rua) foi cerca de 2,43 e 2,29 vezes maior, respectivamente, para os que tinham até 7 anos de estudo quando comparados aos mais escolarizados. Em Santiago, essa diferença foi igual a 1,85 (tabela 4).

\section{Desigualdade social na utilização dos serviços de saúde}

Observamos, na primeira etapa do modelo estimado para a utilização dos serviços ambulatoriais, a ausência de desigualdade social em todas as cidades latino-americanas analisadas. $\mathrm{Na}$ segunda etapa, a educação foi significativa para explicar o número esperado de consultas apenas em Santiago, na Cidade do México e em São Paulo, indicando a presença de desigualdade na quantidade de tratamento recebida por grupos educacionais. Entretanto, o grupo social favorecido dependeu da cidade analisada (tabela 5). Em Santiago e na Cidade do México, constatamos a presença de desigualdade favorável aos idosos de maior escolaridade. O número médio de consultas foi, respectivamente, 81,63 e $29,17 \%$ maior para os idosos com mais de 7 anos de estudo em comparação ao grupo de menor escolaridade. Em São Paulo, o oposto foi observado, ou seja, o número esperado de consultas foi $24,72 \%$ menor para os idosos mais escolarizados. Este resultado surpreende devido ao duplo canal de acesso ao sistema de saúde existente no Brasil, constituindo-se em uma fonte de desigualdade na utilização desses serviços. Os indivíduos que pertencem a classes sociais mais altas podem utilizar os serviços de saúde tanto no setor privado como no setor público, uma vez 
TABELA 5. Utilização dos serviços de saúde por grupo de escolaridade conforme o modelo ambulatorial e o modelo de internação hospitalar estimados para seis cidades latinoamericanas

\begin{tabular}{lccc}
\hline & \multicolumn{2}{c}{ Variável de escolaridade } \\
\cline { 2 - 3 } \multicolumn{1}{c}{ Modelo ambulatorial } & & Modelo de internação hospitalar \\
\cline { 2 - 3 } \multicolumn{1}{c}{ Cidade } & $\begin{array}{c}\text { Segunda etapa: } \\
\text { Primeira etapa: } \\
\text { OR }(\%)\end{array}$ & $\begin{array}{c}\text { melo Negbin truncado } \\
\text { ao zero (\%) }\end{array}$ & OR (\%) \\
\hline Buenos Aires & $39,30^{\mathrm{b}}$ & $-9,25^{\mathrm{b}}$ & $17,52^{\mathrm{b}}$ \\
São Paulo & $-1,99^{\mathrm{b}}$ & $-24,72^{\mathrm{c}}$ & $256,59^{\mathrm{c}}$ \\
Santiago & $27,45^{\mathrm{b}}$ & $81,69^{\mathrm{d}}$ & $74,08^{\mathrm{b}}$ \\
Havana & $4,31^{\mathrm{b}}$ & $2,28^{\mathrm{b}}$ & $31,10^{\mathrm{b}}$ \\
Cidade do México & $23,23^{\mathrm{b}}$ & $29,17^{\mathrm{d}}$ & $51,66^{\mathrm{b}}$ \\
Montevidéu & $29,33^{\mathrm{b}}$ & $13,56^{\mathrm{b}}$ & $-16,92^{\mathrm{b}}$ \\
\hline
\end{tabular}

a Categoria de referência: $\leq 7$ anos de estudo. $O R=$ razão de chances.

b Não significativo.

c Significativo a $5 \%$

d Significativo a $1 \%$.

que, neste último, o acesso é universal, integral e gratuito. Além disso, tal resultado difere daqueles encontrados em outros estudos realizados para o Brasil considerando os demais grupos etários (39). Uma possível explicação é que o custo da oportunidade para procurar cuidados preventivos pode ser mais elevado para os idosos mais escolarizados. Alguns estudos têm mostrado que, no Brasil, as chances de o idoso permanecer no mercado de trabalho depois de aposentado são mais elevadas para os que têm maior nível de escolaridade (40). Nesse sentido, esses indivíduos teriam menos disponibilidade de tempo para procurar cuidados médicos, tendendo a procurar os serviços de saúde apenas quando não é mais possível adiar a realização desse cuidado.

Para o setor de internação hospitalar, os resultados encontrados apontaram a ausência de desigualdades sociais na utilização desses serviços em todas as cidades analisadas. A presença dessa desigualdade foi observada apenas em São Paulo, onde a probabilidade de o indivíduo ser internado foi 3,57 vezes maior entre os idosos mais escolarizados (tabela 5). Esse resultado verificado em São Paulo provavelmente reflete o padrão diferenciado de tratamentos demandados entre os idosos doentes e saudáveis em cada grupo de escolaridade. Os grupos de maior escolaridade, por possuírem também um melhor estado de saúde, têm maiores chances de estar inseridos no mercado de trabalho e, em decorrência disso, procuram menos os serviços preventivos de saúde. Nesse caso, a procura é realizada em situações de emergência, que exigem um tratamento mais intensivo.

Os resultados se mantiveram quando foram incluídas as variáveis "posse de automóvel" e "posse de casa própria". Essas duas medidas não foram significativas para explicar a utilização dos serviços ambulatoriais e de internação hospitalar.

\section{DISCUSSÃO}

A contribuição deste artigo foi diagnosticar a presença da desigualdade social em saúde e na utilização dos serviços desse setor entre os idosos residentes na área urbana de seis cidades da América Latina. Os resultados indicam a presença da desigualdade social em saúde, em todas as cidades analisadas, favorável aos grupos socioeconômicos privilegiados, indo ao encontro dos resultados obtidos para os países desenvolvidos (3-7). Para a maioria das medidas de saúde consideradas, essa desigualdade foi mais acentuada em São Paulo e na Cidade do México, e menor em Buenos Aires, em Havana e em Montevidéu. Esses resultados parecem refletir a desigualdade de renda e as diferenças no nível de desenvolvimento e no processo de transição demográfica verificadas entre esses países. Nas cidades que pertencem a países cujos indicadores sociais são melhores, a desigualdade social em saúde é mais baixa. Além disso, os países se diferenciam segundo o processo de envelhecimento populacional. $\mathrm{Na}$ Argentina, em Cuba e no Uruguai, onde as cidades analisadas possuem as menores desigualdades sociais em saúde, esse processo se encontra em um estágio mais avançado, com uma participação relativamente estável dos idosos na população total. Desse modo, é provável que essas cidades já apresentem uma estrutura mais adequada de atendimento aos idosos.

$\mathrm{O}$ presente estudo mostra que, mesmo em países com sistemas de saúde distintos, com graus diferenciados de cobertura privada e pública, existe desigualdade social em saúde a favor dos mais escolarizados. A investigação da presença de desigualdades sociais na utilização dos serviços de saúde é importante para verificar se as diferenças no estado de saúde entre os grupos de escolaridade estão relacionadas com o acesso ao sistema de saúde. O que se observa é que a desigualdade na utilização dos serviços não foi muito grande, sendo detectada apenas em algumas cidades.

Três possíveis hipóteses podem estar relacionadas a esse resultado. Em primeiro lugar, deve haver algum componente na saúde do idoso, relacionado com o seu estrato social, mas não com a utilização dos serviços, que faz com que se observe a presença de desigualdades sociais em saúde nessas localidades. Esse componente pode estar relacionado com os hábitos e o comportamento do idoso, com a qualidade de vida, a história de vida passada e as características do meio em que o indivíduo está inserido. A segunda hipótese refere-se às diferenças na qualidade dos serviços de saúde entre os idosos mais e menos escolarizados. Ainda que não haja diferenças na utilização dos serviços de saúde, é possível que a qualidade dos serviços 
recebidos pelos idosos menos escolarizados seja inferior, resultando em uma desigualdade social em saúde entre os idosos nessas localidades. Por fim, a terceira hipótese diz respeito ao acesso a outros bens e serviços de saúde, como medicamentos e a contratação de enfermeiros, que deve ser diferenciado entre esses grupos socioeconômicos, podendo resultar em uma menor eficácia do tratamento.

Os resultados obtidos neste trabalho remetem ainda a duas questões. A primeira questão é entender os determinantes da magnitude da desigualdade social em saúde entre diferentes economias. Em particular, encontramos uma desigualdade mais acentuada em São Paulo e na Cidade do México, e menor em Havana, em Buenos Aires e em Montevidéu. As diferenças na magnitude dessa desigualdade podem estar associadas às diferenças no estágio e na velocidade da transição demográfica e nos indicadores socioeconômicos dos respectivos países, sobretudo na distribuição de renda. O Uruguai, a Argentina e Cuba, cujas cidades apresentam as menores desigualdades sociais em saúde, destacam-se por possuírem melhores indicadores socioeconômicos e por estarem em um estágio mais avançado da transição demográfica. $\mathrm{O}$ Brasil e o México, que apresentam os piores indicadores socioeconômicos, com desigualdade de renda elevada e baixo índice de desenvolvimento humano (IDH), são os países onde observamos a desigualdade social em saúde mais elevada, associada à desigualdade social na utilização dos serviços médicos.

Finalmente, cabe indagar se a presença de desigualdade social na utilização dos serviços ambulatoriais está associada ao desenho institucional dos sistemas. Neste trabalho, não verificamos um padrão de relação entre a presença de desigualdade e as características do financiamento e da oferta dos serviços médicos. Por exemplo, entre as cidades nas quais detectamos a ausência de desigualdade social quanto à utilização dos serviços, observamos tanto um sistema de saúde financiado totalmente pelo setor público, como é o caso de Havana, como também um sistema cuja participação do setor privado é predominante, como no Uruguai. Estudos adicionais são necessários nessas e em outras cidades para explicar as interações entre o desenho institucional dos sistemas de saúde e a presença de desigualdades sociais em saúde.

Agradecimentos. As autoras agradecem os comentários preciosos de Luis Rosero, Laura Lídia Rodríguez Wong e dos pareceristas. Agradecemos também à Organização PanAmericana da Saúde (OPAS) e ao Centro de Desenvolvimento e Planejamento Regional (CEDEPLAR), na figura do Professor José Alberto Magno de Carvalho, pelo apoio que permitiu nossa participação nas oficinas de trabalho "Projeto SABE: estudo da saúde do idoso na América Latina" e "Workshop on health and wellbeing of the elderly in Latin America and Caribbean". Agradecemos ainda ao Professor Eduardo Rios Neto, por tornar possível o acesso ao banco de dados utilizado neste trabalho. O presente trabalho foi financiado pela OPAS.

\section{REFERÊNCIAS}

1. Centro Latinoamericano de Demografía (CELADE). Estimaciones y proyecciones de población 1950-2050. Disponível em: http:// www.eclac.cl/celade/proyecciones / intentoBD-2002.htm . Acessado em fevereiro de 2005.

2. Palloni A, Peláez M. SABE: survey on health and well-being of elders: preliminary report. Washington, D.C.: Organización Panamericana de la Salud; 2002.

3. Huisman M, Kunst AE, Mackenbach JP. Socioeconomic inequalities in morbidity among the elderly; a European overview. Soc Sci Med. 2003;57(5):861-73.

4. Smith JP, Kington RS. Race, socioeconomic status, and health in late life. Em: Martin LG, Soldo BJ, eds. Racial and ethnic differences in the health of older Americans. Washington, D.C.: National Academy Press; 1997. Pp. $106-62$.

5. Smith JP, Kington RS. Demographic and economic correlates of health in old age. Demography. 1997;34(1):159-70.

6. Portrait F, Lindeboom M, Deeg D. Health and mortality of the elderly: the grade of membership method, classification and determination. Amsterdã: Free University; 1999. (Research Memorandum Series 1999-22).

7. Portrait F, Lindeboom M, Deeg D. Life expectancies in specific health states: results from a joint model of health status and mortality of older persons. Demography. 2001; 38(4):525-36.

8. Van Ourti T. Socio-economic inequality in illhealth amongst the elderly. Should one use current or permanent income? J Health Econ. 2003;22(2):219-41.

9. Almeida AN. A demanda por serviços de saúde dos idosos no Brasil em 1998. Em: Anais do XIII Encontro da Associação Brasileira de Estudos Populacionais. Disponível em: http://www.abep.nepo.unicamp.br/ docs/anais/pdf/2002/Com_ENV_ST31 Almeida_texto.pdf. Acessado em fevereiro de 2005.

10. Davis K, Reynolds R. Medicare and the utilization of health care services by the elderly. J Hum Resources. 1975;10(3):361-77.

11. Hurd MD, Mcgarry K. Medical insurance and the use of health care services by the elderly. J Health Econ. 1997;16(2):129-54.

12. Miller RH. Access to ambulatory care among noninstitutionalized, activity-limited persons 65 and over. Soc Sci Med. 1992;34(2):1237-47.

13. Filho NA, Kawachi I, Pellegrini A, Dachs N. Research on health inequalities in Latin America and the Caribbean: bibliometric analysis (1971-2000) and descriptive content analysis (1971-1995). Am J Public Health. 2003;93(12):2037-43.
14. Donoso ES. Desigualdad en mortalidad infantil entre las comunas de la provincia de Santiago. Rev Med Chil. 2004;132(4):461-6.

15. Vega J, Bedregal P, Jadue L, Delgado I. Equidad de género en el acceso y financiamiento de la atención de salud en Chile. Disponível em: http://www.puc.cl/equidadchile/html/ genero.pdf . Acessado em fevereiro de 2005.

16. Gómez E. Gender, equality, and health services access: an empirical approximation. Rev Panam Salud Publica. 2002;11(5-6):327-34 .

17. Gakidou E, King G. An individual-level approach to health inequality: child survival in 50 countries. Genebra: World Health Organization; 2000. (Global Programme on Evidence for Health Policy Discussion Paper Series 18).

18. Travassos C, Viacava F, Fernandes C, Almeida CM. Desigualdades geográficas e sociais na utilização de serviços de saúde no Brasil. Cien Saude Col. 2000;5(1):133-49.

19. Almeida C, Travassos C, Porto S, Labra ME. Health sector reform in Brazil: a case study of inequity. Int J Health Serv. 2000;30(1):129-62.

20. Noronha KVMS, Andrade MV. Desigualdades sociais em saúde: evidências empíricas sobre o caso brasileiro. Rev Econ Nordeste. 2002;32:877-97.

21. Campino ACC, Diaz MDM, Paulani LM, Oliveira RG, Piola SF, Nunes A. Country report: Brazil. Em: World Bank. Poverty and eq- 
uity in health in Latin America and the Caribbean: results of country-case studies from Brazil, Ecuador, Guatemala, Jamaica, Mexico and Peru. Washington, D.C.: The World Bank; 1999. Pp. 1-82.

22. Peláez M, Palloni A, Albala C, Alfonso JC, Ham-Chande R, Hennis A, et al. Encuesta salud, bienestar y envejecimiento, 2000. Washington, D.C.: Organización Panamericana de la Salud/Organización Mundial de la Salud; 2003.

23. Palloni A. Protocol of the multicenter study: health, well-being and aging in Latin America and the Caribbean. Washington, D.C.: Pan American Health Organization; 1999. (Technical Papers Series Research in Public Health 6).

24. Lebrão ML, Duarte YAO, orgs. SABE—saúde, bem-estar e envelhecimento: o projeto SABE no Município de São Paulo: uma abordagem inicial. Brasília: Organização Pan-Americana da Saúde; 2003.

25. Wooldridge JM. Econometric analysis of cross section and panel data. Cambridge, MA: MIT Press; 2002.

26. Salomon JA, Tandon A, Murray CJL. Comparability of self rated health: cross sectional multi-country survey using anchoring vignettes. Br Med J. 2004;328(7434):258.

27. Grossman M. On the concept of health capital and the demand for health. J Polit Econ. 1972; 80(2):223-55.

28. Palloni A, Devos S, Peláez M. Aging in Latin America and the Caribbean. Wisconsin: Cen- ter for Demography and Ecology, University of Wisconsin; 1999. (Working Paper 99-02).

29. Szekely M, Hilgert M. What drives differences in inequality across countries. Washington, D.C.: Inter-American Development Bank; 2000. (Research Department Working Paper Series 439).

30. Programa das Nações Unidas para o Desenvolvimento. Relatório de desenvolvimento humano 2002: aprofundar a democracia em um mundo fragmentado. Brasília: PNUD; 2002.

31. Programa de las Naciones Unidas para el Desarrollo. Informe sobre desarrollo humano en la Provincia de Buenos Aires. Buenos Aires: PNUD; 1998.

32. Programa das Nações Unidas para o Desenvolvimento. Atlas de desenvolvimento humano no Brasil (IDH-M), 1991-2000. Brasília: PNUD; 2003.

33. Programa de las Naciones Unidas para el Desarrollo. Investigación sobre el desarrollo humano y equidad en Cuba, 1999. La Habana: PNUD; 2000.

34. Programa de las Naciones Unidas para el Desarrollo. Informe sobre desarrollo humano en México, 2002. PNUD; 2003.

35. Programa de las Naciones Unidas para el Desarrollo. Desarrollo humano en Uruguay, 2001. PNUD; 2001.

36. Organización Panamericana de la Salud. La salud en las Américas. Washington, D.C.: OPS; 1998. (Publicación Científica 569, vol. II).
37. Fleury S, Belmartino S, Baris E, eds. Reshaping health care in Latin America: a comparative analysis of health care reform in Argentina, Brazil, and Mexico. Ottawa: International Development Research Centre; 2000.

38. Tamez S, Molina N. Reorganizing the health care system in Mexico. Em: Fleury S, Belmartino S, Baris E, eds. Reshaping health care in Latin America: a comparative analysis of health care reform in Argentina, Brazil, and Mexico. Ottawa: International Development Research Centre; 2000. Pp. 153-81.

39. Noronha KVMS, Andrade MV. Social inequality in the access to healthcare services in Brazil. Belo Horizonte: Centro de Desenvolvimento e Planejamento Regional, Universidade Federal de Minas Gerais; 2002. (Textos para discussão CEDEPLAR/UFMG td172). Disponível em: http://www.cedeplar.ufmg. $\mathrm{br} /$ pesquisas/td/TD\%20172.pdf. Acessado em fevereiro de 2005.

40. Liberato VC. A oferta de trabalho masculina "pós-aposentadoria" no Brasil urbano1981/2001 [dissertação de mestrado]. Belo Horizonte: Centro de Desenvolvimento e Planejamento Regional, Universidade Federal de Minas Gerais; 2003.

Manuscrito recebido em 29 de junho de 2004. Aceito em versão revisada em 3 de dezembro de 2004.

ABSTRACT Objective. To learn if there is social inequality in health and in the utilization of health services among the elderly in six cities of Latin America: Buenos Aires, Argentina; São Paulo, Brazil; Santiago, Chile; Havana, Cuba; Mexico City, Mexico; and Montevideo, Uruguay.

\section{Social inequality in health and the utilization of health services among the elderly in Latin America} Methods. This study used data from a project called Health, Well-being, and Aging in Latin America and the Caribbean (known as the "SABE project"). To investigate the presence of social inequality in health an ordinal probit model was used, with health status indicated by the following dependent variables: activities of daily living, instrumental activities of daily living, physical mobility, and self-rated health. To measure social inequality in the utilization of health services two characteristics were assessed: one for outpatient medical services (whether or not individuals received outpatient care, and how many times), and one for hospital admissions (whether or not individuals were hospitalized). For outpatient services, we estimated a negative binomial hurdle model, and for hospital admissions, a logit model was constructed.

Results. Our main results suggest the presence of social inequality in health in all the cities, with better-off socioeconomic groups having better health. The difference in health was less marked in Havana, Buenos Aires, and Montevideo, and it was more pronounced in São Paulo and Mexico City. With respect to the utilization of health services we found inequalities in the use of outpatient services in Santiago, Mexico City, and São Paulo. In Santiago and Mexico City, more schooling was associated with a higher number of expected outpatient medical visits; however, the opposite was found in São Paulo. For hospital admissions, inequality was found only in São Paulo, with individuals with more years of schooling being much more likely to be hospitalized.

Conclusion. To a certain extent, our results reflect the socioeconomic and demographic characteristics of the countries in which each of the six cities is located. The cities in the countries with the worst social indicators (high income inequality and low human development index) tended to have the greatest inequalities in health and in the utilization of health services.

Keywords Educational status, health services. 